\title{
Coulisses
}

Revue de théâtre

5 | Hiver 1992

Varia

\section{Antoine Vitez (20 décembre 1930-30 avril 1992)}

\section{Marlène Sainte-Marie Perrin}

\section{OpenEdition}

\section{Journals}

Édition électronique

URL : http://journals.openedition.org/coulisses/1724

DOI : $10.4000 /$ coulisses. 1724

ISSN : 2546-9460

\section{Éditeur}

Presses universitaires de Franche-Comté

\section{Édition imprimée}

Date de publication : 1 janvier 1992

Pagination : 38-43

ISSN : 1150-594X

\section{Référence électronique}

Marlène Sainte-Marie Perrin, «Antoine Vitez (20 décembre 1930-30 avril 1992) », Coulisses [En ligne],

5 | Hiver 1992, mis en ligne le 04 juillet 2017, consulté le 22 octobre 2019. URL : http:// journals.openedition.org/coulisses/1724 ; DOI : 10.4000/coulisses.1724

Ce document a été généré automatiquement le 22 octobre 2019

Coulisses 


\title{
Antoine Vitez (20 décembre 1930-30 avril 1992)
}

\author{
Marlène Sainte-Marie Perrin
}

Le 6 avril 1991 a eu lieu la traditionnelle cérémonie de la remise des Molière au cours de laquelle Redjep Mitrovitsa, Molière du meilleur acteur de l'année précédente, pour son interprétation de Lorenzaccio, trophée qu'il avait dédié à son professeur et metteur en scène, lit un texte d'Antoine Vitez, un texte où il est question des difficultés de mémorisation. Au cours de la même soirée, Dominique Valadié qui reçoit le Molière de la meilleure actrice parle avec chaleur et authenticité d'Antoine Vitez.

2 Le lundi 30 avril 1990 au matin, mourait Antoine Vitez, frappé d'une hémorragie cérébrale ; il avait 59 ans, renouant dans une étrange constellation littéraire avec ceux qu'il avait servis : Brecht mort à 59 ans, après les répétitions de La Vie de Galilée. Claudel qui, a 59 ans, en février 1927, quittait le Japon, sa dernière étape orientale, à bord du Korea Maru, pour rejoindre les États-Unis. Deux ans plus tôt il avait terminé Le Soulier de satin, vision baroque et apaisée de sa rupture avec cette Ysée du Partage de midi, qu'il avait rencontrée sur un autre bateau, l'Ernest Simon alors qu'il rejoignait pour la seconde fois la Chine.

3 Si jouvre le programme du Soulier de satin et que je lis la distribution: Anne Benoit, Elisabeth Catroux, Valérie Dréville, Jany Gastaldi, Philippe Girard, Serge Maggiani, Madeleine Marion, Daniel Martin, Ludmila Mikhaël, Redjep Mitrovitsa, Alexis Nitzer, Aurélien Recoing, Robin Renucci, Didier Sandre, Dominique Valadié, Pierre Vial, Gilbert Vilhon, Jeanne Vitez, Judith Vitez, Jean-Marie Whiling, il suffit d'ajouter quelques noms : Patrice Kerbrat, Michel Aumont, Nadia Strancar, Richard Fontana et Roland Bertin - pour obtenir le noyau de ceux qui ont travaillé avec lui ; amis fidèles dont certains ont été ses élèves et qui se souviennent de son enseignement généreux et de l'amour qu'il leur portait, de son exigence faite d'authenticité et de rigueur, cette rigueur que certains de ses détracteurs ont confondu avec cérébralité, intellectualisme désincarné. Or il suffisait de s'asseoir et d'assister au spectacle pour se convaincre du contraire. 
4 J'ai gardé de ces soirées de Chaillot une série d'images, des voix qui ne me quitteront plus tel un album de contes feuilleté dans l'enfance et qui sont aussi vrais que les choses vécues dans ces premières années. Vitez ne disait-il pas : « Si je suis devenu metteur en scène c'est que j'ai désiré mettre en œuvre des choses que j'avais envie de voir ». Le désir était le moteur de son travail, l'aiguillon de son défi: "je me provoque moimême, il n'est pas possible que je n'arrive pas à le faire si j'ai vraiment envie de le voir ».

5 On retrouve cette même rigueur, cette exigence chez Anne Delbée et Sophie Loukachevsky, deux de ses anciennes élèves du Conservatoire où il eut sa classe de 1976 à 1981 avant de fonder sa propre école au Théâtre national de Chaillot : on lui donna la salle Louis Jouvet. On peut penser qu'il y fut sensible, lui qui admira Louis Jouvet sans jamais l'approcher et qui, tout comme lui, porta sur le théatre de Molière un regard aigu et cruel, le délivrant ainsi du jeu poussiéreux et codé que l'on a bien souvent réservé aux « Classiques ».

6 Pour ceux qui n'auront vu aucune de ses mises en scène, il restera les enregistrements de son travail, les entretiens qu'il accorda et ils seront sans doute frappés, comme nous aujourd'hui, par la qualité de son expression. Car l'exigence de la langue était chez Vitez une exigence de tous les instants, il suffisait de l'entendre pour être sous le charme de cette langue précise. Il n'y avait aucune montre d'un quelconque narcissisme mais bien la preuve d'une rigueur, celle du poète, du traducteur et du metteur en scène qui étaient en lui : il lui arrivait souvent de dire une phrase et de la reprendre tout aussitôt en des termes différents par souci du mot juste et afin d'être bien compris de tous. De cette juxtaposition de mots surgissait la musique, musicalité de la langue qui lui faisait préférer en traducteur-poète Sophocle à Euripide : il goûtait la perfection éclairée de Sophocle comme il goûtait une composition de Bach. Cet amour de la langue belle et juste l'amenait parfois à des traductions controversées parce que dépoussiérées, dans leur sens premier, ainsi celle de La Mouette: "J'adore faire des traductions et la mise en scène commence à la traduction. Je la fais la plus libérale possible comme une version latine. Au lieu de chercher comme mes prédécesseurs une plus grande fluidité, j'ai respecté la lourdeur volontaire et parfois l'ordre des mots en russe. Je n'ai pas cherché à être plus malin que Tchékhov ».

7 Cet incroyant avait pour livre de chevet Les Pensées de Pascal et L'Apocalypse selon Saint Jean. Ses lectures avaient un lien secret avec ce rôle qui nous restitue une image de ce qu'il fut: le rôle du marxiste pascalien Vidal, professeur de philosophie dans Ma nuit chez Maud. On dit que Rohmer réécrivit le rôle après avoir engagé Vitez et l'avoir écouté. Revanche sans doute pour celui que Vilar engagea comme accessoiriste, pigiste de sa revue, puis comme comédien mais à qui il ne donna jamais aucun rôle car, lui ditil, «Si les seigneurs sont les comédiens, les rois eux doivent attendre ». Mais laissons les arguments de la légende et analysons ce travail dont nous cherchons à notre tour par cet article à conserver la trace brillante.

8 Dans son travail, le sensuel, volontiers facétieux qui, avant une répétition préparait les acteurs par des jeux de mots, l'imitation d'une personnalité ou d'une voix, se doublait d'un archéologue : "Ce qui m'intéresse au théâtre c'est l'éternité, la chair humaine, reconstituée à travers les générations ». Travail d'où le plaisir intellectuel de voir passer le temps n'était sans doute pas exclu, et qui consistait d'abord à connaître l'histoire de la pièce qu'il allait monter: une biographie détaillée de son auteur, une connaissance précise de moment de son écriture, les différentes mises en scène faites 
jusque là : travail d'étude et d'assimilation nécessaire pour aller de l'avant . Certains entretiens donnés alors que La Mouette se jouait à Chaillot illustrent précisément cela : il donne son avis sur les mises en scène de La mouette par Meyerhold puis par les Pitoëff avant d'aborder son propre travail. Il avait fait sienne cette phrase de Meyerhold: "Chacun de nos spectacles a un double but: ce qu'il faut faire pour le public, et ce qu'il faut faire pour que nous progressions ».

9 Je dirais pour ma part que Vitez dans ce qu'il a apporté au théâtre s'est conduit en archéologue qui dégage les soubassements de l'édifice, ces soubassements indispensables au travail de l'acteur afin de lui donner les moyens de jouer et de remplir sa mission. Pour cela il a veillé tout d'abord à rétablir la diction. Il s'y emploie dès ses premières mises en scène puis, lorsqu'une classe lui est donnée au Conservatoire National, il fait de la diction un des éléments importants de la formation. Grâce à lui, une génération d'acteurs a retrouvé cette manière juste et musicale de dire le vers, qu'il s'agisse de Racine, de V. Hugo ou de Claudel... Déclamation encore décriée par certains mais qui permet de rendre son jeu premier, son existence a des pièces lues mais réputées injouables, comme je l'entendais dire autour de moi quand je lisais le théâtre de Victor Hugo. Hernani à Chaillot m'a permis de retrouver l'enthousiasme adolescent, les images qui avaient accompagné les premières lectures. Un texte que l'on entend dans sa musicalité éveille l'imaginaire du spectateur et participe de la volonté du théâtre qui doit parfois suggérer plus qu'il ne montre.

Vitez avait commencé ce travail avec Racine, à travers cette musicalité de l'alexandrin : accents, pauses, silences. Vitez rétablissait la vérité poétique et prouvait que le langage plus que véhicule de l'idée, était le corps lui-même. Malgré cette éblouissante résurrection du corps au théâtre, certains n'ont cessé de voir en lui un cérébral. Ce goût de l'alexandrin chez Racine, son goût des mythes grecs et son désir de retrouver « les corps et leurs jeux entre eux et avec leurs voix dans le lit du théâtre l'a poussé à mettre en scène un texte oublié, superbe de force, de violence non retenues, bien supérieur par sa sauvagerie à celui de Phèdre de Racine empreint d'une grandeur policée : Hippolyte de Garnier à la salle Gémier en 1982 ; et toute sa mise en scène a consisté à montrer les forces monstrueuses de la pièce. Le dénouement violent m'est encore très présent : je revois Phèdre qui se jette sur le corps d'Hippolyte mort, dans une impossible et amoureuse possession.

11 Après Racine vient la confrontation avec le vers claudélien; si, pour Vitez Sophocle vaut une partition de Bach, Le Soulier de satin est au théâtre ce que Le Sacre du printemps est à la musique. Cette approche musicale de l'œuvre, Claudel y consent lorsqu'il présente les quatre personnages de L'Échange comme quatre chants qui se répondent. Pour Vitez l'écriture de Claudel est une partition: «La présentation typographique de Claudel oblige l'intonation de l'acteur, c'est-à-dire qu'on ne peut échapper à la typographie de Claudel, parce que sa typographie est une notation exactement comme la notation d'une musique sur une partition.»

Donc sans avoir besoin d'écrire des notes sur une portée, Claudel par le système même de son écriture, par la coupe des mots, la coupe des vers, par l'introduction des expressions toutes faites crée un système d'intonation obligée du vers. Vitez dit par ailleurs que «si l'on suivait ce rythme du vers on retrouvait le parler rocailleux et paysan de l'homme Claudel, son souffle ».

13 Tout ce prodigieux travail fait d'amour et d'intuition est au service de l'acteur, pièce maîtresse de cette révolution du théâtre, l'acteur qu'il n'a eu de cesse de révéler à lui- 
même, de conduire jusqu'au bout de lui-même afin qu'il donne au public étonné des personnages de chair et de sang faits pour l'interroger et le bouleverser : « La cause du théâtre ? C'est celle des comédiens. Ce sont les images qu'ils font naître qui permettent aux spectateurs d'avoir des rapports privilégiés avec le texte : ce sont leurs visages qui laissent des traces, il est donc essentiel de laisser l'acteur se construire une œuvre en continu dans la mémoire du public... L'acteur doit étonner, poser des questions ; jouer naturel c'est-à-dire comme à la télévision, c'est endormir le spectateur dans une image plate dans laquelle il est censé se reconnaître, c'est en fait le modeler sournoisement ; il faut donc jouer au-delà du vrai. Le travail du comédien doit s'attacher à détruire toutes les tentations de l'illusion, à libérer tous les clichés qui l'emprisonnent ».

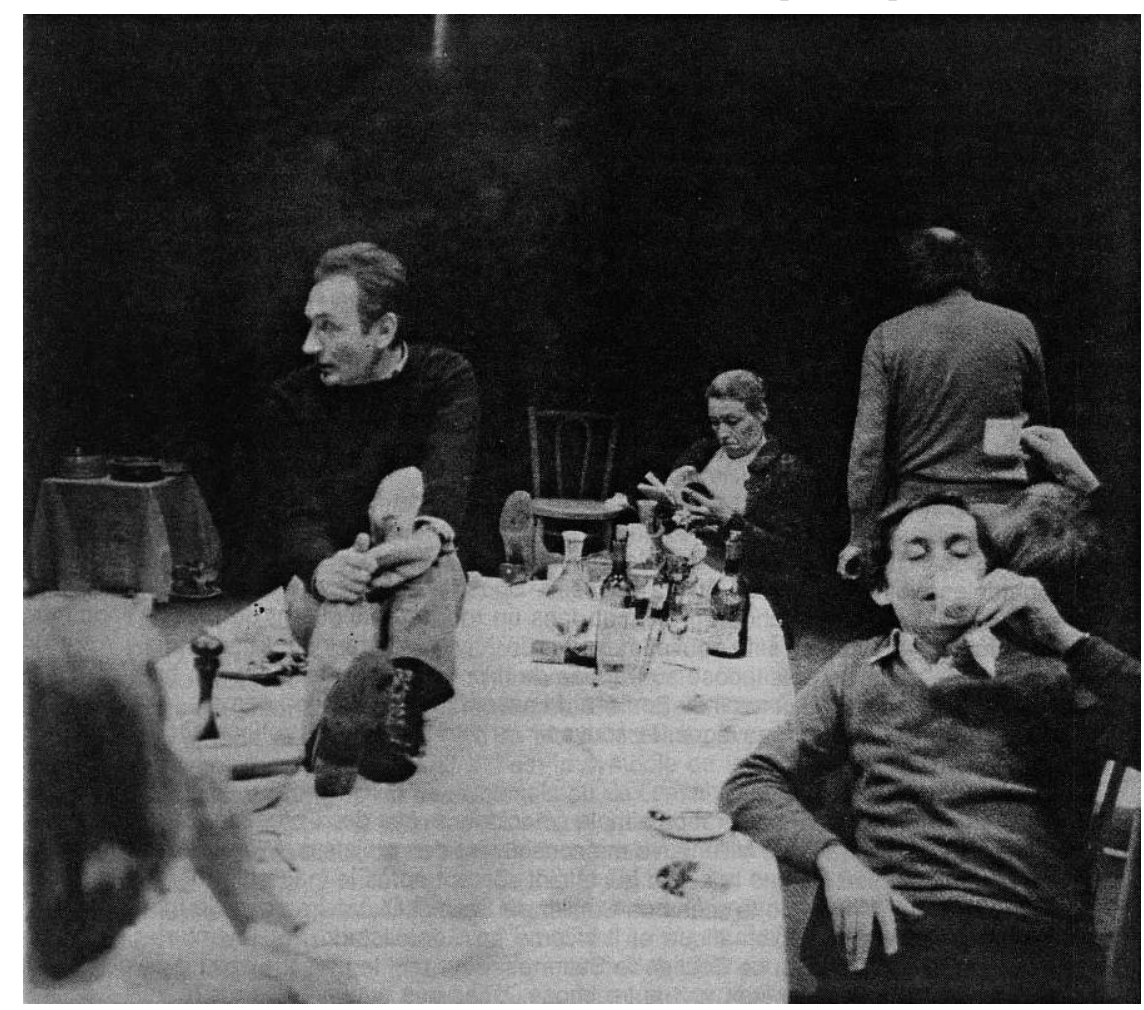

Antoine Vitez pendant une pause, in Avignon, 40 ans de festival, Hachette, p. 139

Conjointement à cette direction d'acteurs plus proche de l'accouchement que de la dictature, Ludmila Mikhaël déclare dans un entretien au sujet du Soulier: «sans sa grande tolérance, sans son grand respect de la fatigue de l'acteur nous serions peutêtre morts. Il voit tout, il entend tout, mais il n'empiète pas sur l'imagination de l'acteur ». Didier Sandre a expliqué bien souvent sa surprise et ses difficultés lors du premier Misanthrope lorsqu'il s'aperçut que Vitez ne cherchait pas à le diriger, mais essayait de le conduire, l'obligeant à une réceptivité nouvelle. Le travail de l'espace et de la lumière joue un rôle essentiel: Vitez sent très rapidement le jeu dans l'espace scénique et c'est à l'acteur de s'y conformer dès le début des répétitions comme témoigne Richard Fontana qui interpréta Hamlet en 1983 : «il laisse une très grande liberté dans un cadre hyper précis : il sait trouver les places et les déplacements. Parfois on ne comprend pas et puis soudain tout s'éclaire, on ne peut pas être ailleurs ». La scène nue est celle de l'imaginaire allié à la rigueur, il confie lui-même à propos de son travail sur La Célestine : "À la différence par exemple de Strehler qui ne peut travailler en répétition que dans les décors et les costumes de la pièce, sur la scène où elle sera jouée, je pars du principe exactement contraire, selon lequel il faut préparer en 
gommant les accessoires, les objets pour mieux les imaginer, pour mieux aussi les désirer. » des réussites. Je me souviens du Britannicus et de l'agacement que je ressentis à la sortie ; sous l'échec vivait le spectacle tel qu'il aurait dû être. Tous les acteurs n'avaient pas sauvé leur mise mais Jean Baptiste Malartre, Jeanne Vitez et Claude Durand nous avaient donné, à travers ce qu'ils nous laissaient entrevoir, le dépit de ce que nous avions manqué. Le Faust qui suivit le Britannicus me laisse aussi une impression mitigée mais plus forte. Jamais je ne suis sortie de Chaillot avec le sentiment d'avoir perdu mon temps car chaque pièce s'ajoutait aux autres, comme un rêve, une exigence intérieure, un chemin qu'il voulait montrer aux autres et cela spectacle après spectacle nous le sentions, tout comme nous sentions sa générosité.

Le Soulier de satin, magistral aboutissement du long compagnonnage avec Claudel, qu'Aragon et Madeleine Marion lui firent aimer - présenté en juillet 1987 dans la cour 
des Papes à Avignon, est fort de toute sa générosité envers cet homme si différent de lui mais dont il a tout compris : « je ne suis pas chrétien, je ne crois pas à la vie éternelle, mais je suis claudélien au sens où l'entendait le poète: si on respecte sa prose, ses césures, son rythme, alors on met le pas dans son souffle ». Cette intuition généreuse va jusqu'à accepter sans le partager les options de l'autre car le chrétien sans faille vit le même absolu que le révolutionnaire : «Et quand au début de la $3^{\mathrm{e}}$ journée, on parle de l'extermination des protestants, ce langage est soutenu par une foi si puissante, qu'un communiste, même s'il a été victime du système, peut le comprendre ».

"Le théâtre doit aussi servir à construire une Cité modèle », et c'est au nom de cette exigence qu'il se servait de sa vie, des ses interrogations privées et publiques pour saisir sous le texte vieux de soixante ans ou de trois siècles une autre réalité plus proche de la nôtre - sous l'Espagne des Conquistadors il voit le colonialisme de la III République - et lui donne par là même un surcroît de vie : "Je me suis aperçu que tous ceux qui comme moi, ont participé au mouvement communiste, dont je me suis toujours senti proche, sont des gens qui ont rêvé le monde, cela se passe dans Le Soulier de satin.»

Cette exigence d'un théâtre qui serait le miroir d'une Cité idéale le pousse à monter trois fois en vingt ans avec la même comédienne - Evelyne Istria dans le rôle titre Électre en intercalant des «parenthèses » de Yannis Ritsos. Le décor de Yannis Kokkos souligne la pérennité des mythes avec l'histoire: le palais patricien qui s'ouvre sur le port grec d'aujourd'hui. Électre est le symbole de la résistance au tyran, qu'il s'agisse du dictateur antique ou des Colonels ou de l'envahisseur nazi car l'enjeu de la pièce est de montrer comment certains s'adaptent au compromis et comment pour d'autres la vie n'a de valeur qu'au nom de la mémoire et de la liberté quel qu'en soit le prix. Électre est une réflexion sur la Cité et la Démocratie car «d'une manière générale, le théâtre a perpétuellement des leçons à donner aux politiques ", «il anticipe sur la pensée civique ". Sa dernière mise en scène La vie de Galilée à l'heure où le bloc socialiste s'effondre, tout comme sa lecture en juillet 1989 du Discours sur le colonialisme d'Aimé Césaire à Avignon ou les mises en scène plus anciennes comme Iphigénie-Hôtel de Michel Vinaver ou Le Procès d'Emile Henry, spectacle-montage écrit par lui-même sont, avec d'autres pièces, le parcours le plus évident de cette exigence.

Pour finir, ces quelques phrases auxquelles le vide donne un tout autre sens.

Ce que je sais, je suis forcé de le dire à d'autres. Comme un amoureux, comme un ivrogne, comme un traître. C'est un vice absolu, et il conduit au malheur. Combien de temps vais-je pouvoir le crier dans le noir ? Telle est la question. 